\title{
SIMULATION OF SURFACE-TYPE CONDENSING UNITS FOR HEAT RECOVERY FROM THE FLUE GAS WITH AIR HEATING
}

\author{
Victor V. Bespalov ${ }^{1,{ }^{*}, \text { Leonid A. Beljaev }}{ }^{1}$, and Leonid S. Kuchman ${ }^{1}$ \\ ${ }^{1}$ Tomsk Polytechnic University, 634050, Tomsk, Russia
}

\begin{abstract}
The technology of flue gas heat recovery has been reviewed. The mathematical model requirements were analyzed and determined. The mathematical model of the installations, that implement this technology, was developed and described in paper. As a result, the software application that implements a mathematical model has allowed to design the different installations and can be useful to design organizations in the energy industry.
\end{abstract}

\section{Introduction}

The increased interest of industrial and energy enterprises in energy saving defines the development and introduction of new technologies.

The flue gas heat recovery from the natural gas combustion can provide an additional $15 \div 20 \%$ of the boiler thermal capacity [1] or, respectively, reduce the gas flared amount. Scientists develop the recovery technologies and improve the heat transfer calculation methodologies with water vapor condensation contained in the flue gases [1-3]. The technology developed allows to increase the flue gas heat recovery depth through the use air as a heated medium [4, 5]. For designing the installations, that implement this technology, are required the mathematical model that allows to carry out the thermal and constructional calculations of the entire system, including the component parts. The mathematical model must include the heat balance calculations of elements and the heat transfer calculations through the heat exchange surfaces. The balance equations in joint solution allow to find the thermal power of the elements and the flows temperatures. The heat transfer equations associate the thermal power of each element with its heat exchange area.

The proposed installation scheme (Fig. 1) [4] consists of a recuperative heat exchanger and condensing heat recovery unit. Gas-gas surface plate heat exchanger provides precooling the initial flue gas and simultaneous heating cooled and dried flue gas in order to prevent possible condensation of residual moisture in the flues and chimney. Heat recovery unit (gas-air surface plate heat exchanger) constructive single device is conventionally divided into two parts: cooler (dry zone) and the condenser. Air is fed to the condenser by

\footnotetext{
*Corresponding author: vic@tpu.ru
} 
fan unit. Additional exhauster compensates aerodynamic resistance of the gas path in the heat exchanger and condenser.

\section{Mathematical model}

The mathematical model requires to preset the heat exchangers geometry. The heat exchanger and the heat recovery unit are compiled of the heat exchange modules (packages). The sheet length, width and thickness, the thermal conductivity coefficient, the number of sheets and the clearance between them are determined for each package. The number of packages and heat-carrier passages are set for each heat exchanger. The package geometry can be different for heat exchanger and heat recovery unit, however, for highpower installations (more than $1 \mathrm{MW}$ ) it is advisable to use the unified packages. So, the unified package has been developed for power installation $10 \mathrm{MW}$ (flue gas flow $33 \mathrm{~m}^{3} / \mathrm{s}$ ), consisting of 72 sheets, size $2500 \times 1250 \mathrm{~mm}$ and weight about 1.2 tons [4]. The number of packages for such a flue gas flow amounted to 16 for the heat exchanger and 24 for the heat recovery unit.

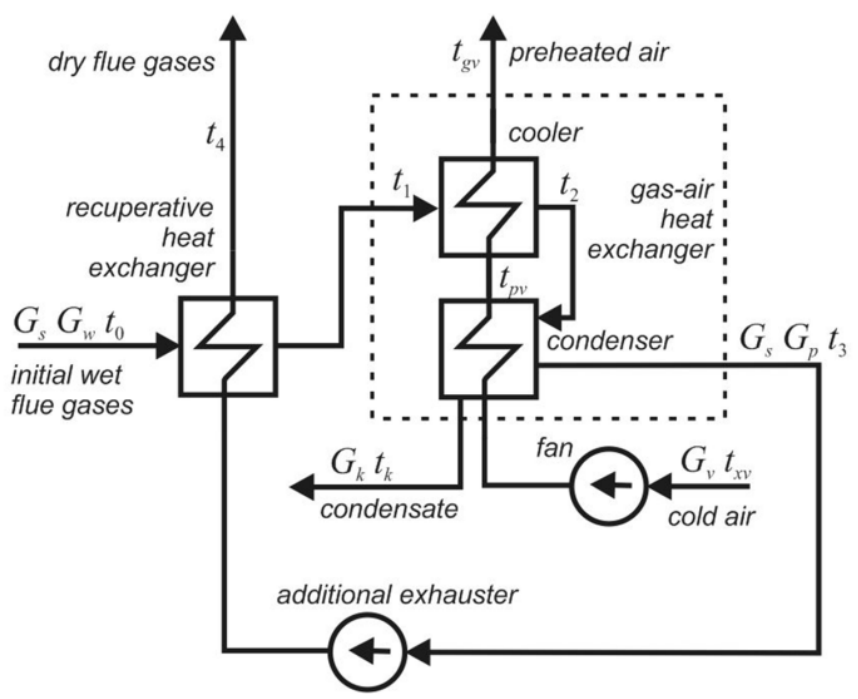

Fig. 1. The technology of flue gas deep heat recovery.

The input data for the mathematical model are the cold air temperature $t_{x v}$ and flue gas mass flow consisting of a dry flue gas $G_{s}$ and water vapor $G_{w}$ with the temperature $t_{0}$. The flue gas outlet temperature $t_{2}$ from cooler assumes to be equal to the water vapor dew point at the initial humidity. Other parameters are calculated by successive approximations. A block diagram of the model structure is shown in Figure 2.

Block 1 contains the source data input and setting the initial values of the unknown parameters in the first approximation. This block also calculates the heat transfer surface area in all the elements $F_{p}, F_{t}$ and the equivalent diameter of passage sections in the heat exchangers. The flue gas temperature at the condenser outlet $t_{3}$ is defined in a first approximation as a minimum (close to $0^{\circ} \mathrm{C}$ for negative air temperatures).

Block 2 is designed to calculate the flue gas composition $G_{s}, G_{w}$ and expected condensate flow $G_{k}$. The heat transfer coefficient $k_{t p}$ for heat exchanger is set in the first approximation, based on the gas flow velocity, and will be further refined by the simple iterations method. 
Block 3 calculates the thermal balance of the recuperative heat exchanger. The log means temperature difference is defined by as a first approximation and refined by the dichotomy method.

Block 4 calculates a heat transfer process in the recuperative heat exchanger and finds the corrected value (next approximation) of heat transfer coefficient $k_{t p}$. If the relative error of the next approximation exceeds $0.1 \%$ then there is a return to block 3 . Otherwise, it is considered that heater has been conditionally calculated at a specified flue gas temperature at the outlet of the condenser $t_{3}$.

Block 5 calculates the thermal balance in the cooler and condenser to determine the air temperatures $t_{g v}$ and $t_{p v}$. The heat transfer coefficient $k_{t o x}$ for cooler is found from heat transfer equations and then the dry zone area can be calculated.

Block 6 calculates the condensation zone heat transfer process. From the air flow side the heat transfer coefficient depends on the Reynolds number. The main difficulty is determining the heat transfer coefficient $\alpha_{1}$ from a wet flue gas to the heat exchange surface with the water vapor condensation. Most of the known methods are based on experimental data processing and offer the dependences in rather narrow limits changes in the physical parameters. Applicability of a calculation method for functionally similar heat exchangers requires analysis and study [6]. Reviewed model allows to choose different methods to calculate the heat transfer coefficient from wet flue gas to the heat exchange surface.

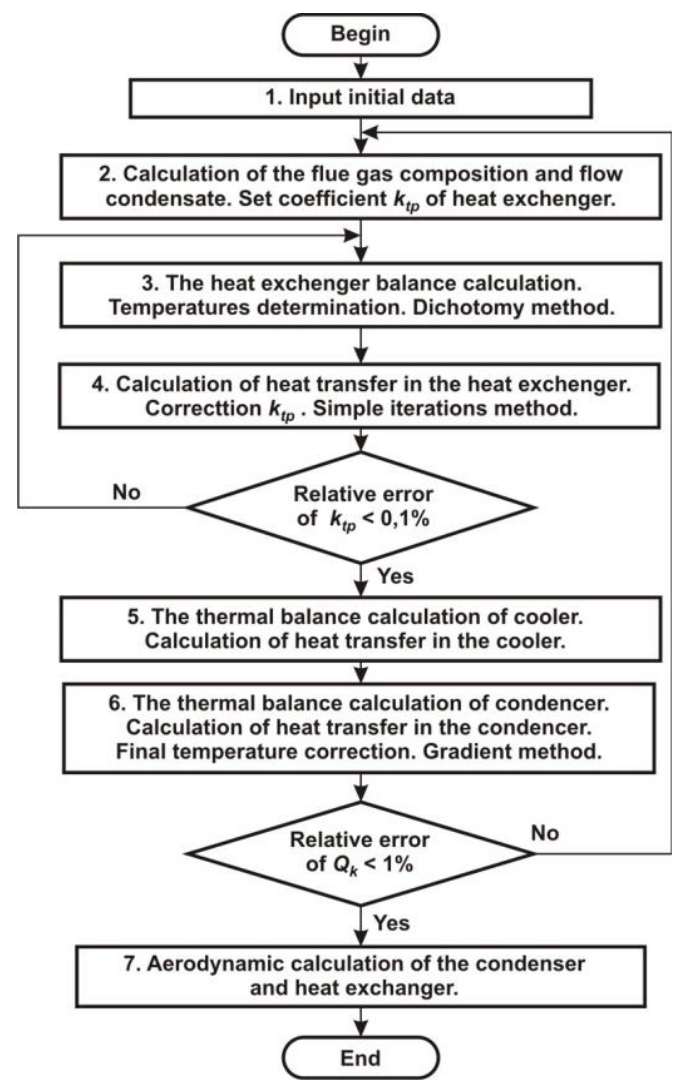

Fig. 2. A block diagram of the mathematical model structure.

Condenser power capacity $Q_{k}$ is calculated by the heat transfer equation. If it is less the same power calculated from the balance equations, the final flue gas temperature at the 
condenser outlet $t_{3}$ is adjusted upwards by $0.01{ }^{\circ} \mathrm{C}$, or downward by the same amount. Then return to the block 2 . The algorithm prevents the transition to negative temperatures with the message about the condensate freezing possibility at the given parameters. The outer loop terminates when the thermal power capacity relative error lower $1 \%$. This cycle implements the gradient method. Other methods do not give a sustainable solution because the results are high sensitive to parameters value change.

Block 7 completes the necessary calculations. The pressure losses of air and flue gas are calculated for the heat recovery unit and the heat exchanger.

\section{Results}

The developed mathematical model is implemented as a software application that allows to choose the optimal geometric parameters of the installations for various capacities and verify their work.

A number of installations were designed by the calculations results [7]. The calculations were performed for the average temperature of the winter months in Siberia. At lower temperatures, the heat recovery will be more deep and the final flue gas temperature at the condenser outlet will decrease. The initial flue gas temperature is sufficiently high, resulting in a large dry zone in the heat recovery unit, reaching up to $45 \%$ of heat exchange area. This decreases the condensation zone and reduces the heat recovery depth.

When installing the heat recovery unit is expedient to reduce the flue gas temperature at the boiler outlet to $100{ }^{\circ} \mathrm{C}$ by embedding additional surface for heating water in the boiler flue. This will increase the recovery depth and will reduce the final flue gas temperature below $20^{\circ} \mathrm{C}$.

\section{Conclusion}

A mathematical model of the condensing plate-type heat recovery unit using air as a heated medium contains the necessary heat balance equations and the heat transfer equation with selection different calculation methods. Equation systems are solved by various numerical methods that provide acceptable result accuracy.

The software application that implements a mathematical model allows to design the different installations and carry out verification calculations. The application will be useful to design organizations in the energy industry.

\section{References}

1. A. Kudinov, V. Antonov, Y. Alekseev, Thermal Engineering, 47, 65 (2000).

2. K. Jeong, M. Kessen, H. Bilirgen, E. Levy, International Journal of Heat and Mass Transfer, 53, 2361 (2010).

3. A. Garjaev, E. Tseplyaeva, Sur l'efficacite energetique, 1, 187 (2003).

4. V. V. Bespalov, Power engineering of Tatarstan, 38, 32 (2015).

5. V. V. Bespalov, L. Beljaev, D. Melnikov, MATEC Web of Conferences, 37, 01009 (2015)

6. V. V. Bespalov, V. I. Bespalov, D. Melnikov, EPJ Web of Conferences, 110, 01007 (2016)

7. V. V. Bespalov, Power engineering of Tatarstan, 42, 39 (2016) 